\title{
Correction to: Multiple endocrine neoplasia type 1: analysis of germline MEN1 mutations in the Italian multicenter MEN1 patient database
}

\author{
Francesca Marini ${ }^{1}$ - Francesca Giusti ${ }^{1}$ - Caterina Fossi ${ }^{1} \cdot$ Federica Cioppi $^{1}$ - Luisella Cianferotti ${ }^{1}$ - Laura Masi $^{1}$. \\ Francesca Boaretto ${ }^{2}$. Stefania Zovato ${ }^{2}$ - Filomena Cetani $^{3} \cdot$ Annamaria Colao $^{4}$ - Maria Vittoria Davi ${ }^{5}$. \\ Antongiulio Faggiano ${ }^{4}$ Giuseppe Fanciulli ${ }^{6}$ Piero Ferolla ${ }^{7}$. Diego Ferone ${ }^{8} \cdot$ Paola Loli $^{9} \cdot$ Franco Mantero $^{10}$. \\ Claudio Marcocci ${ }^{3}$. Giuseppe Opocher ${ }^{2}$ - Paolo Beck-Peccoz ${ }^{11}$ - Luca Persani ${ }^{12,13}$ - Alfredo Scillitani ${ }^{14}$. \\ Fabiana Guizzardi $^{13} \cdot$ Anna Spada $^{12} \cdot$ Paola Tomassetti $^{15} \cdot$ Francesco Tonelli $^{1} \cdot$ Maria Luisa Brandi ${ }^{1}{ }^{1}$
}

Published online: 21 July 2018

(c) Springer Science+Business Media, LLC, part of Springer Nature 2018

\section{Correction to: Endocrine 2018}

https://doi.org/10.1007/s12020-018-1566-8

The original version of this article unfortunately contained a mistake in Table 2. The Table 2 was truncated in the original publication. The full Table 2 is given below.

The original article has been corrected.

Maria Luisa Brandi

marialuisa.brandi@unifi.it

1 Department of Surgery and Translational Medicine, University of Florence, Florence, Italy

2 Familial Cancer Clinic, Veneto Institute of Oncology IRCCS, Padua, Italy

3 Department of Clinical and Experimental Medicine, Section of Endocrinology, University of Pisa, Pisa, Italy

4 Endocrinology Unit, Department of Clinical Medicine and Surgery, University of Naples Federico II, Naples, Italy

5 Internal Medicine, Section of Endocrinology, Department of Medicine, University of Verona, Verona, Italy

6 NET Unit, Department of Clinical and Experimental Medicine, University of Sassari, Sassari, Italy

7 Multidisciplinar NET Center, Umbria Regional Cancer Network, Azienda Ospedaliera di Perugia and University of Perugia, Perugia, Italy
8 Endocrinology Unit, Department of Internal Medicine and Medical Specialties (DiMI), Policlinico San Martino, University of Genoa, Genoa, Italy

9 Department of Endocrinology, Hospital Niguarda Ca' Granda, Milan, Italy

10 Division of Endocrinology, Department of Medicine (DIMED), University of Padua, Padua, Italy

11 University of Milan, Milan, Italy

12 Department of Clinical Sciences and Community Health, University of Milan, Milan, Italy

13 Division of Endocrine and Metabolic Diseases, IRCCS Istituto Auxologico Italiano, Milan, Italy

14 Unit of Endocrinology 'Casa Sollievo della Sofferenza' Hospital, IRCCS, San Giovanni Rotondo, Foggia, Italy

15 Department of Medical and Surgical Sciences, S. Orsola-Malpighi University Hospital, Bologna, Italy 


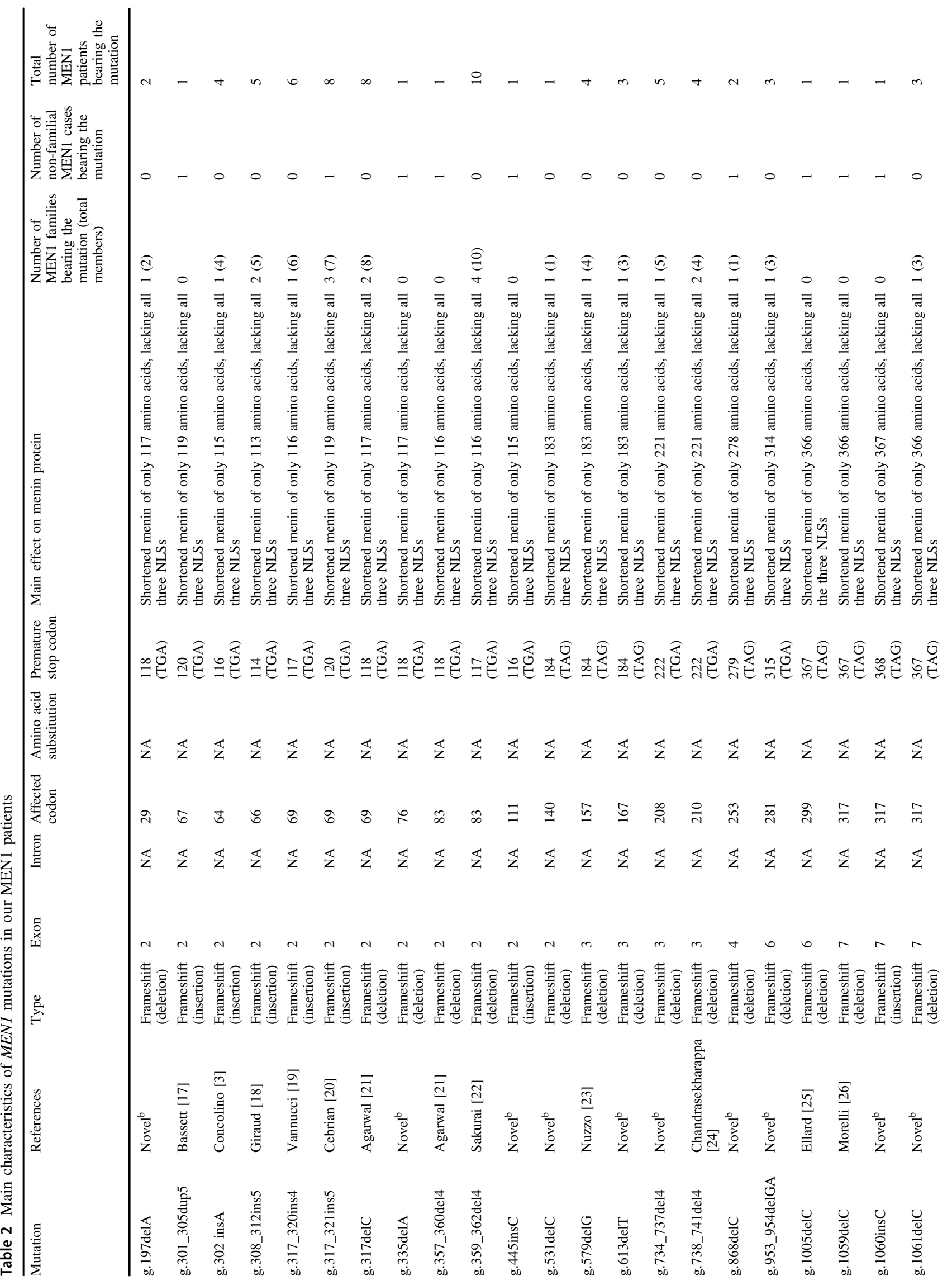




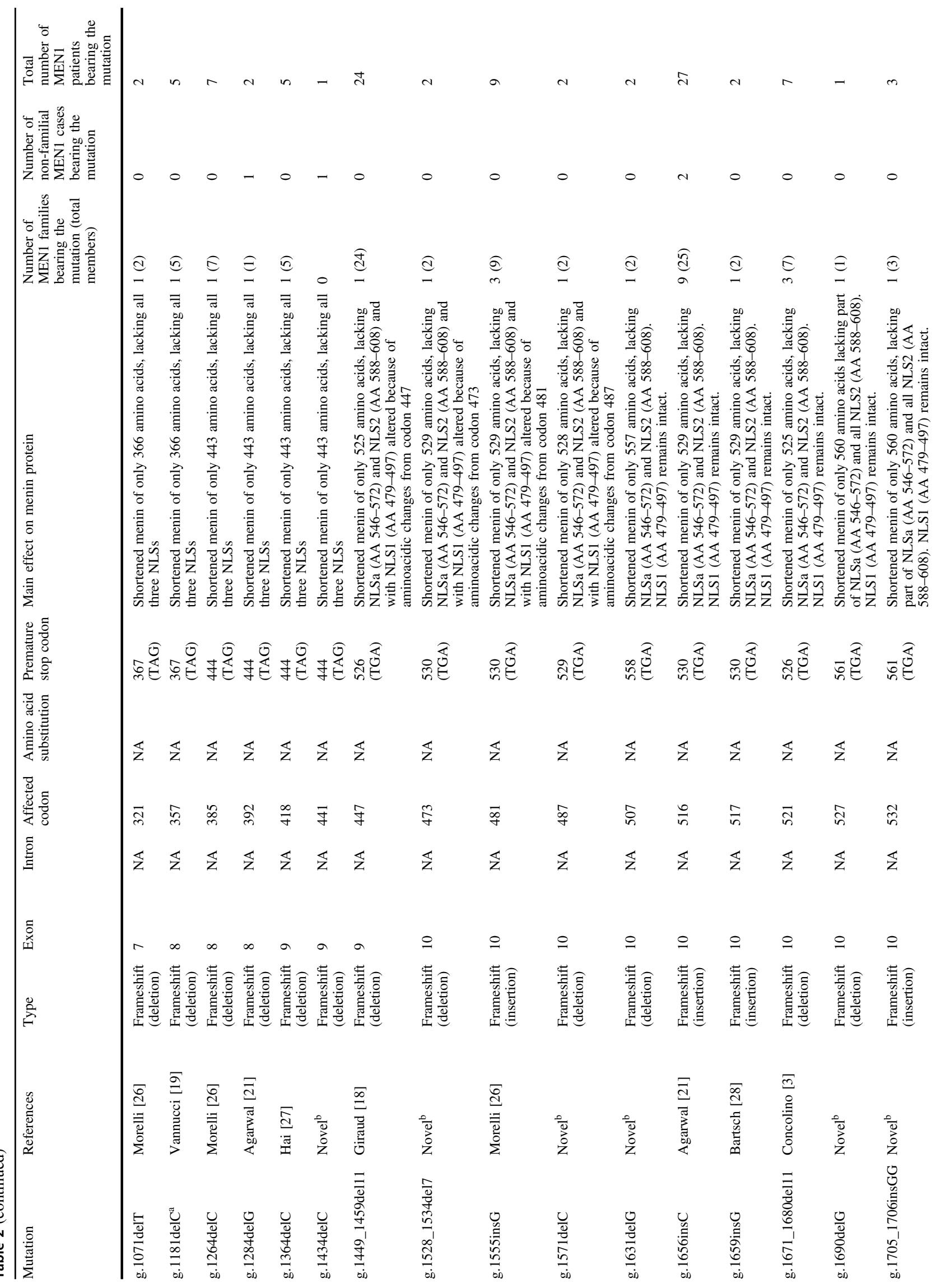




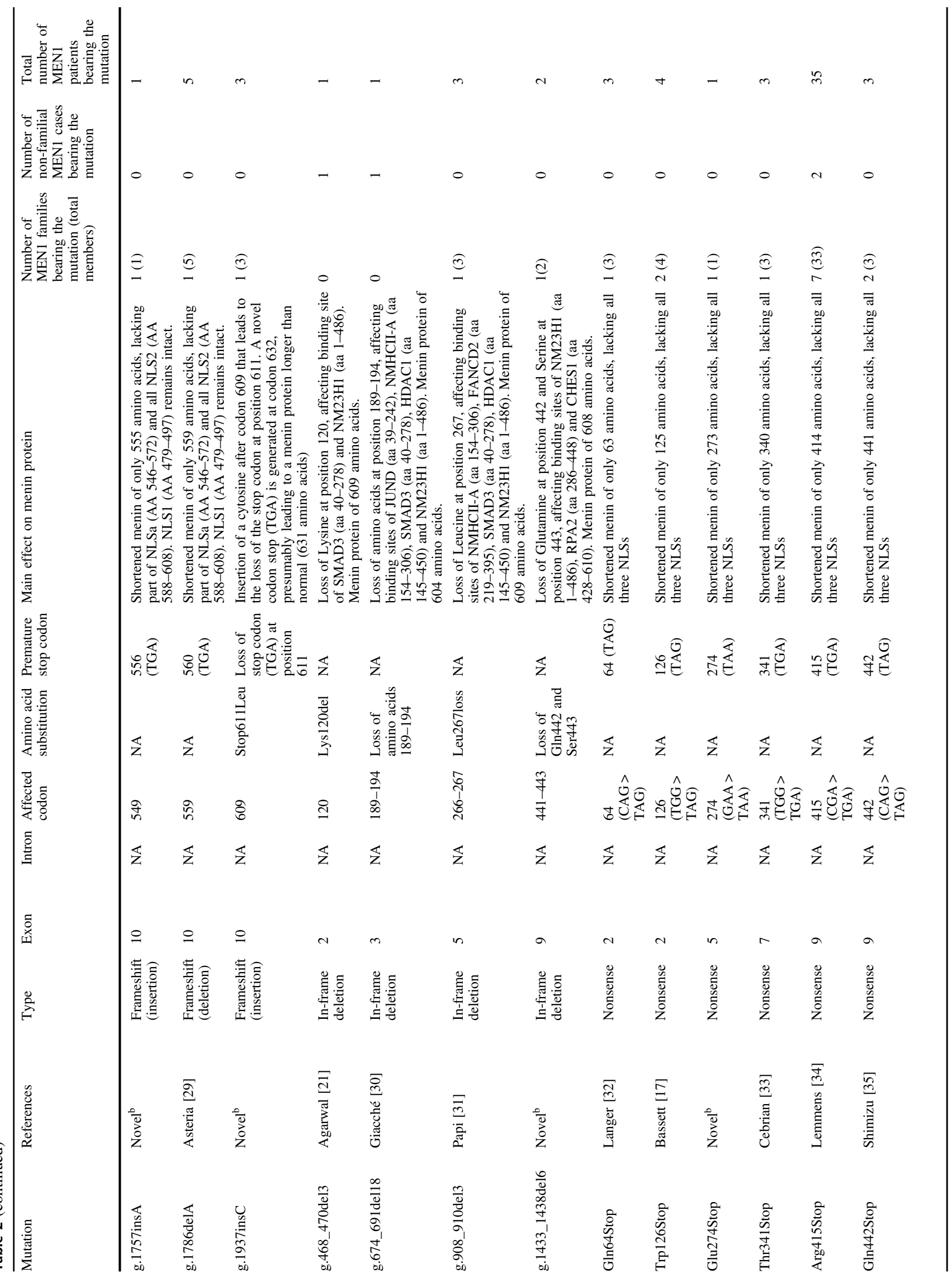




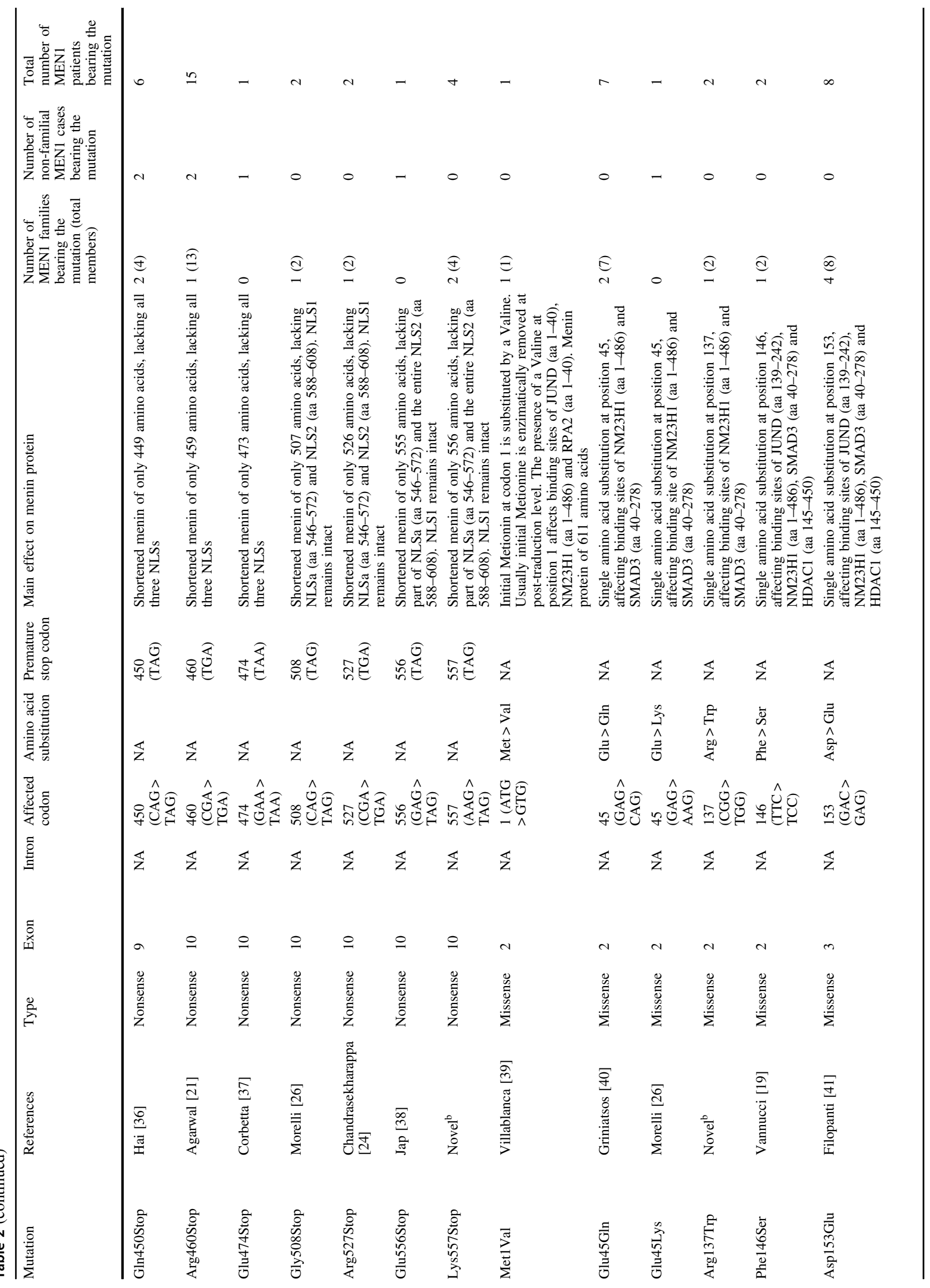




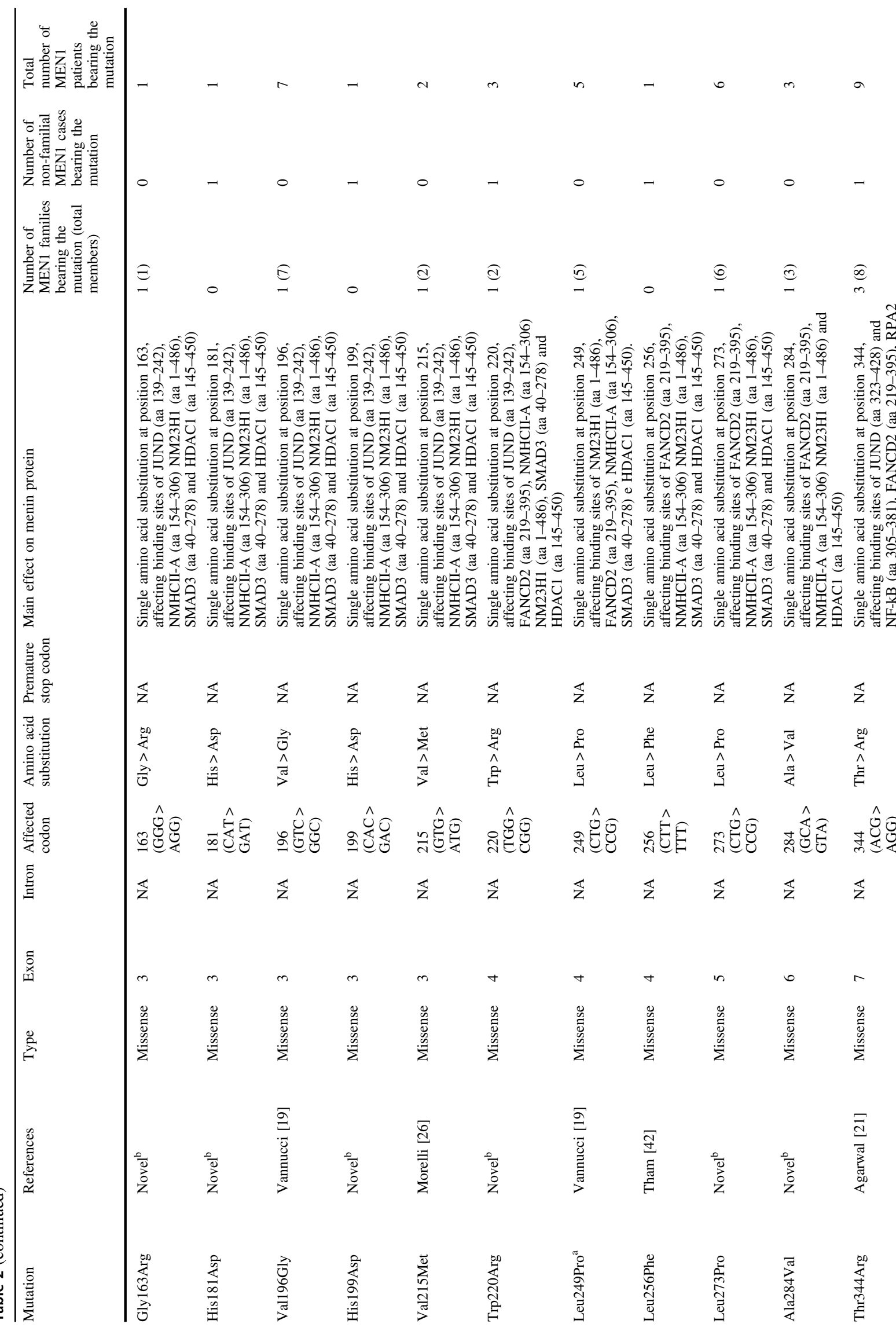




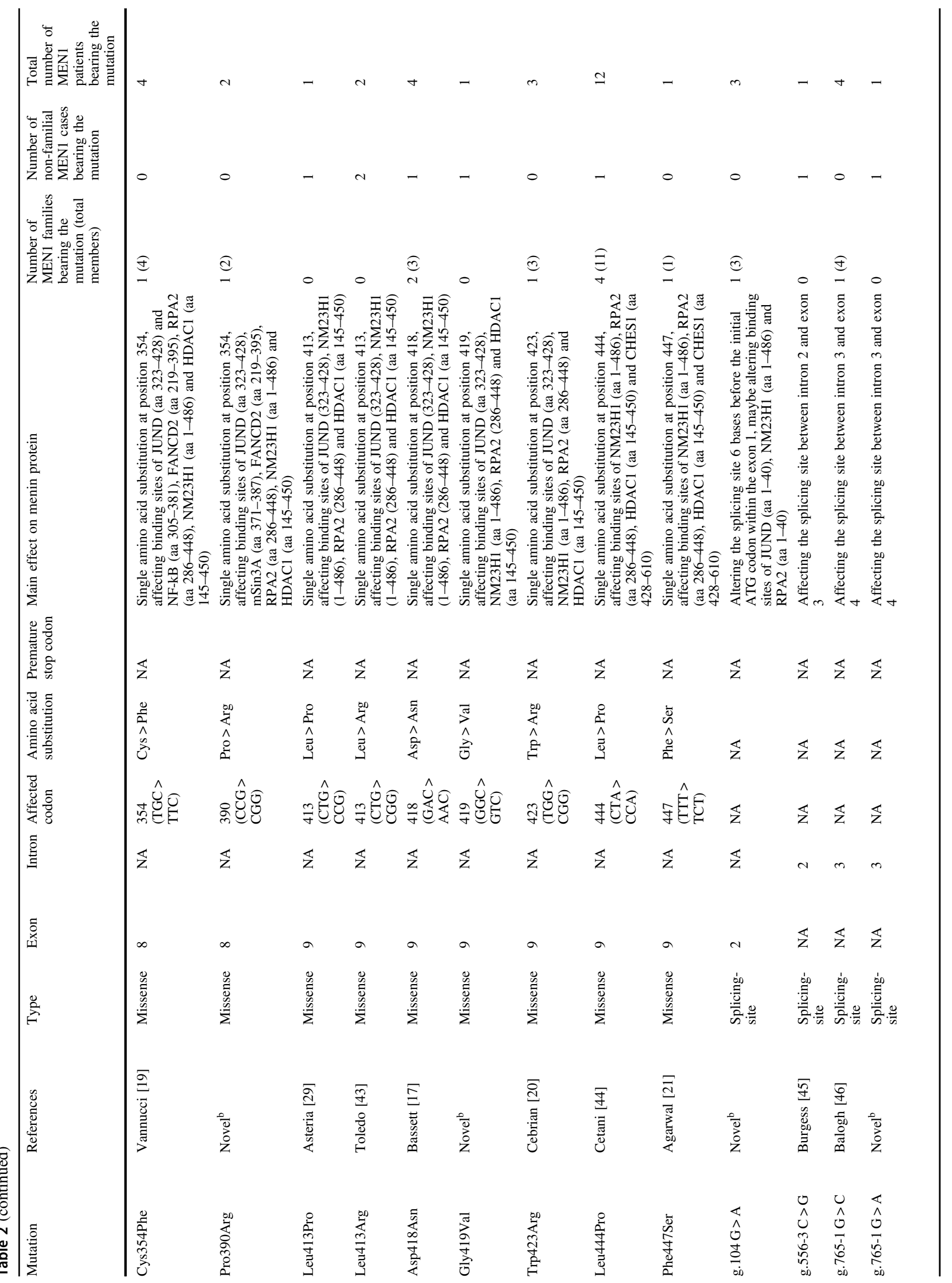




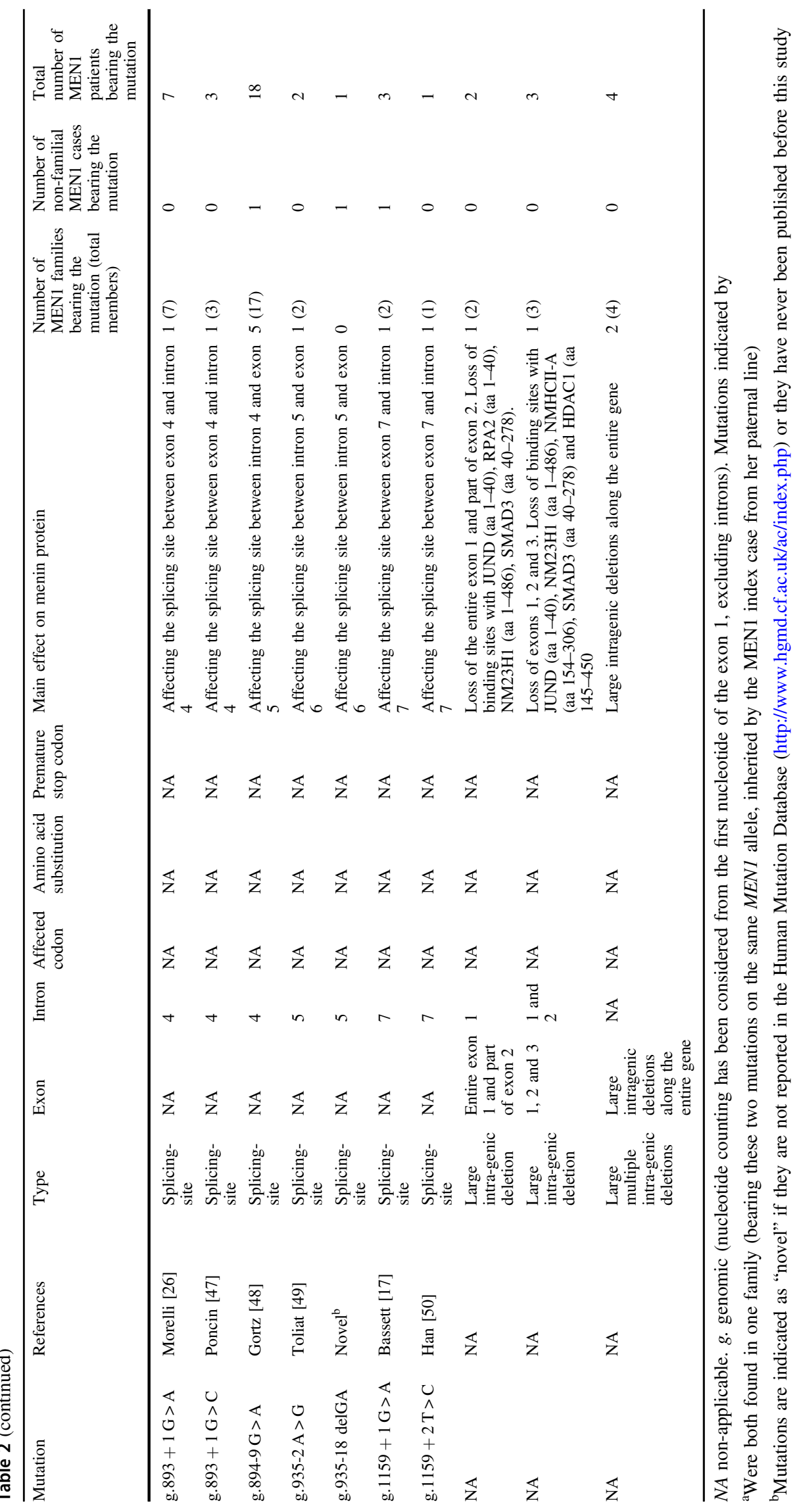

